\section{Early colon cancer presenting as intussusception and successfully treated using endoscopic submu- cosal dissection}

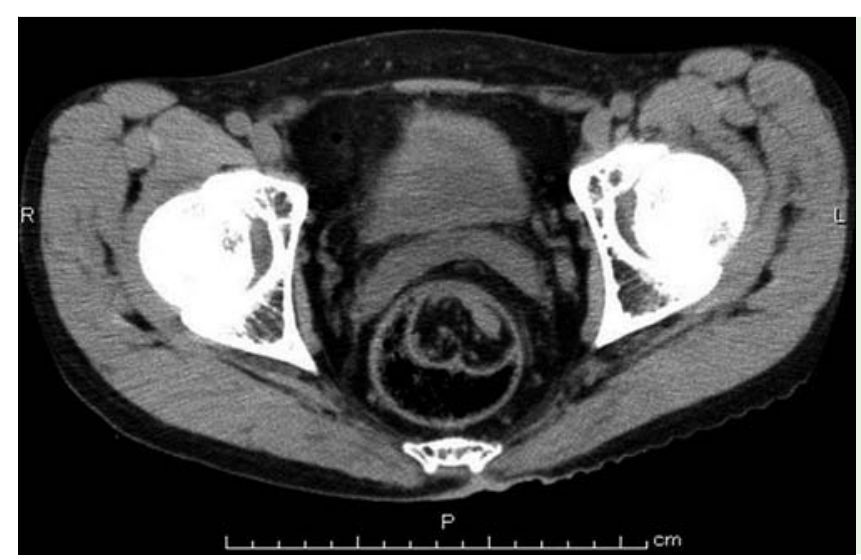

Fig. 1 Computed tomographic appearance of intestinal intussusception caused by a sigmoid tumor in a 57-year-old man, showing the characteristic soft-tissue target sign.

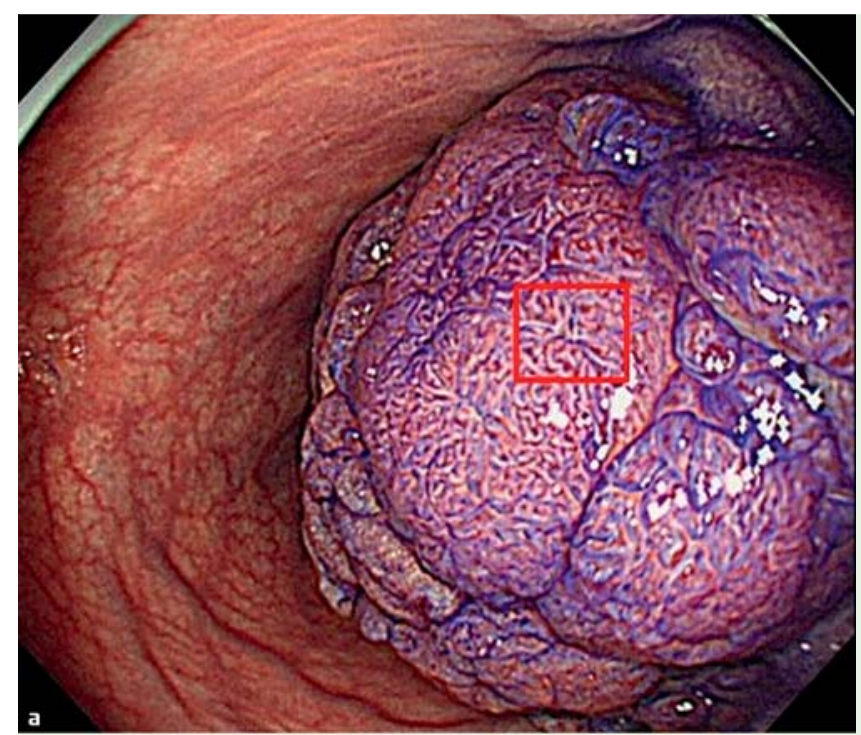

Fig. 2 a Standard endoscopic view with crystal violet staining. b Magnified view with crystal violet staining (red square in a). We diagnosed these findings as consistent with the type VI pit pattern.
Adult intussusception represents $5 \%$ of all cases of intussusception, and accounts for $1 \%$ of all cases of bowel obstruction [1]. Due to the significant risk of malignancy associated with this condition, with neoplasia found in approximately $65 \%$ of cases [2], intussusception in adults is treated by surgery, and in most cases cancer is discovered intraoperatively [3]. Thus, in most cases adult intussusception requires treatment by definitive surgical resection [2]. We report the first case of adult intussusception caused by early sigmoid colorectal cancer, which was successfully resected endoscopically.

A 57-year-old man was admitted to our hospital with the complaints of lower abdominal pain and a tumor prolapsing through the anus. His white cell count was $8100 / \mu \mathrm{L}$ and his blood hemoglobin level $12.4 \mathrm{~g} / \mathrm{dL}$. Abdominal computed tomography showed intestinal intussusception caused by the sigmoid tumor ( Fig. $\mathbf{1}$ ). The tumor was manually reduced into the rectum, relieving the patient of pain. The following day, sigmoidoscopy revealed a large subpedunculated polyp measuring about $50 \mathrm{~mm}$ in diameter. Magnifying endoscopy with crystal violet staining (৫ Fig.2) did not suggest that the tumor was associated with massive invasion of the submucosal layer, and endoscopic submucosal dissection (ESD) was performed. During this procedure, severe fibrosis and muscle retraction were observed in the submucosal layer ( $\bullet$ Fig.3); however, en bloc resection was successfully performed without any complications. The tumor was $50 \times 40 \mathrm{~mm}$ in size; histological examination revealed intramucosal carcinoma, and that all the margins were tumor-free (๑ Fig.4).

Several reports have described an endoscopic treatment approach for intussusception associated with benign tumors $[4,5]$. However, there have been no reports of endoscopic resection carried out in cases of intussusception caused by early colorectal cancer. In our case, the physical findings, CT findings, and precise endoscopic diagnosis [6] after reduction of the prolapsing tumor led us to an accurate diagnosis, allowing this large tumor to be resected not by surgery, but by cautious ESD, which is a minimally invasive treatment [7].

Endoscopy_UCTN_Code_CCL_1AD_2AB

Competing interests: None 


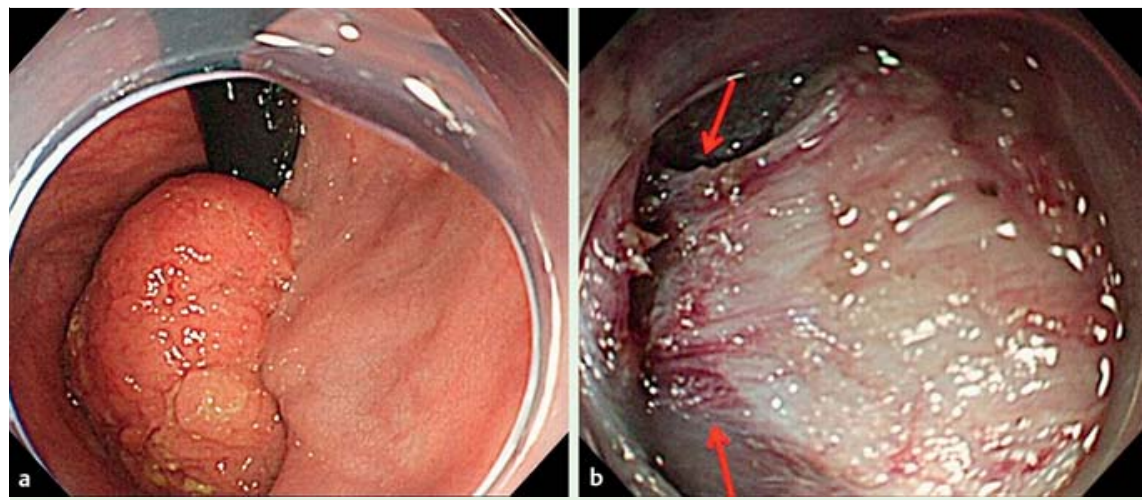

Fig. 3 a Endoscopic view before the start of endoscopic submucosal dissection (ESD). First, the tumor and its margin were carefully inspected. $\mathbf{b}$ During ESD, the muscle layer was observed to be retracted into the submucosa (red arrows).

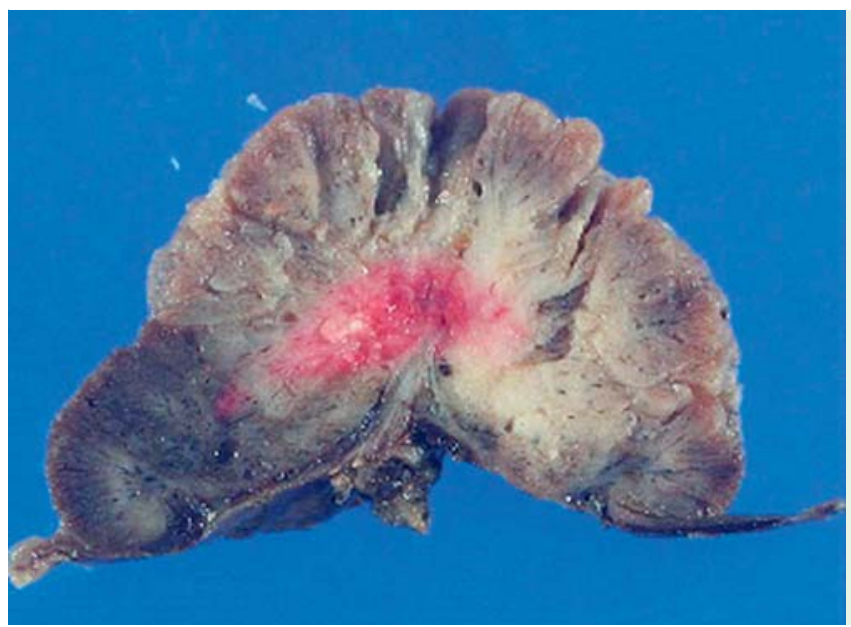

specimen after formalin fixation, showing a retracted muscle layer.
Hideyuki Chiba', Akihiro Takahashi', Masahiko Inamori², Toru Goto ${ }^{1}$, Ken Ohata ${ }^{3}$, Nobuyuki Matsuhashi ${ }^{3}$, Atsushi Nakajima

${ }^{1}$ Department of Gastroenterology, Omori Red Cross Hospital, Tokyo, Japan

${ }^{2}$ Gastroenterology Division, Yokohama City University Hospital, Kanagawa, Japan ${ }^{3}$ Department of Gastroenterology, NTT Medical Center Tokyo, Tokyo, Japan

\section{References}

1 Azar T, Berger DL. Adult intussusception. Ann Surg 1997; 226: $134-138$

2 Begos DG, Sandor A, Modlin IM. The diagnosis and management of adult intussusception. Am J Surg 1997; 173: 88-94

3 Akcay MN, Polat M, Cadirci M et al. Tumorinduced ileo-ileal invagination in adults. Am Surg 1994; 60: 980-981

4 Yoshimura H, Murata K, Takase Ket al. A case of lipoma of the terminal ileum treated by endoscopic removal. Gastrointest Endosc 1997; 46: 461 - 463

5 Lin BC, Lien JM, Chen RJ et al. Combined endoscopic and surgical treatment for the polyposis of Peutz-Jeghers syndrome. Surg Endosc 2000; 14: 1185-1187

6 Kudo S, Hirota S, Nakajima T et al. Colorectal tumours and pit pattern. J Clin Pathol 1994; 47: $880-885$

7 Ohata K, Nonaka K, Minato $Y$ et al. Endoscopic submucosal dissection for large colorectal tumor in a Japanese general hospital. J Oncol 2013; 2013: 218670

\section{Bibliography}

Dol http://dx.doi.org/

10.1055/s-0034-1377224

Endoscopy 2014; 46: E326-E327

(c) Georg Thieme Verlag KG

Stuttgart · New York

ISSN 0013-726X

Corresponding author

Hideyuki Chiba

4-30-1, Chuo, Ota-Ku

143-8527 Japan

Tokyo

h.chiba04@gmail.com 